José Marçal Jackson Filho ${ }^{\text {a* }}$

iD https://orcid.org/0000-0002-4944-5217

Ada Ávila Assunção ${ }^{\text {b* }}$

(iD) https://orcid.org/0000-0003-2123-0422

Eduardo Algranti ${ }^{\text {** }}$

(iD) https://orcid.org/0000-0002-6908-7242

Eduardo Garcia Garcia ${ }^{\S}$

(iD) https://orcid.org/0000-0002-4300-4314

Cézar Akiyoshi Saito ${ }^{\S}$

(iD) https://orcid.org/0000-0002-4931-1884

Maria Maeno ${ }^{\mathrm{c} \pm}$

(iD) https://orcid.org/0000-0001-6329-629X

a Fundação Jorge Duprat Figueiredo de Segurança e Medicina do Trabalho, Unidade Descentralizada do Paraná.

Curitiba, PR, Brasil.

b Universidade Federal de Minas Gerais, Faculdade de Medicina, Programa de Pós-graduação em Saúde Pública. Belo Horizonte, MG, Brasil.

' Fundação Jorge Duprat Figueiredo de Segurança e Medicina do Trabalho, Centro Técnico Nacional. São Paulo, SP, Brasil.

*Editor(a) Chefe da RBSO

$\S$ Editor Executivo da RBSO

${ }^{ \pm}$Editora Associada da RBSO

Contato:

José Marçal Jackson Filho

E-mail:

jose.jackson@fundacentro.gov.br

\section{A saúde do trabalhador e o enfrentamento da COVID-19}

\author{
Worker's health and the struggle against COVID-19
}

O enfrentamento da pandemia do novo coronavírus faz parte das funções essenciais da Saúde Pública por meio de ações voltadas para a população ou para grupos com maior risco de contaminação, como os profissionais de saúde. No entanto, outras atividades de trabalho podem ter um papel relevante na disseminação do vírus e, portanto, a análise de como se processam é determinante para a prevenção do adoecimento ${ }^{1}$. A pouca visibilidade desse aspecto implica sua pouca valorização nas políticas públicas. O campo do trabalho como um todo deve ser considerado na estratégia de enfrentamento da COVID-19.

No início do surto, na cidade de Wuhan na China, trabalhadores e clientes de um mercado atacadista de frutos do mar, considerado como o provável foco inicial da contaminação pelo manuseio de animais vivos, estiveram entre os primeiros casos de pessoas infectadas (cerca de $55 \%$ dos 47 casos reportados até o dia $1^{\circ}$ de janeiro de 2020) ${ }^{2}$. Em Singapura, $68 \%$ dos 25 casos iniciais de contaminação comunitária foram atribuídos ao exercício profissional $^{3}$. No Brasil, o segundo óbito por coronavírus registrado foi o de uma empregada doméstica no Rio de Janeiro, cuja doença foi contraída no exercício do trabalho ${ }^{4}$.

Essas situações, a exemplo de outras, mostram que tanto o exercício das atividades laborais quanto as condições de trabalho são fontes potenciais de exposição ao vírus. Por sua vez, esse lócus - a situação de trabalho é território de disseminação da doença ${ }^{1}$. É fundamental entender, portanto, de que maneira as atividades e condições de trabalho podem contribuir para a disseminação e, sobretudo, para o estabelecimento de estratégias para o enfrentamento da pandemia.

Mesmo para os profissionais de saúde diretamente envolvidos com os cuidados aos pacientes, pouco se discute sobre as condições e organização do trabalho, prevalecendo, até o momento, protocolos com recomendação de medidas individuais (higiene e uso de equipamentos de proteção) ${ }^{5}$, fundamentais, mas insuficientes para o controle geral da disseminação e da exposição ao vírus. Todas as medidas de proteção previstas no protocolo de manejo clínico do coronavírus, no Brasil, dizem respeito à biossegurança ${ }^{6}$. Mas há relatos de profissionais e sindicatos denunciando condições de trabalho precarizadas, higiene inadequada, jornadas extenuantes, falta de treinamento e, inclusive, insuficiência ou indisponibilidade de equipamentos de proteção, mesmo nos serviços de terapia intensiva ${ }^{7}$. Em estudos sobre a gripe influenza, observou-se a interferência de vários fatores na adesão às práticas de biossegurança, entre eles, sobrecarga de trabalho, precariedade das instalações e normas de convívio social ${ }^{8}$. Na África, durante a epidemia do Ebola, as recomendações sanitárias se mostraram contraditórias com as práticas de cuidado dos agentes comunitários de saúde, que relataram constrangimento ao evitar aperto de mãos, recusar água, café ou outro alimento, ou ainda, não aceitar o convite para sentar durante as visitas de acompanhamento da quarentena ${ }^{9}$. Na China, houve a criação de serviços on-line de 
acompanhamento da saúde mental e aconselhamento psicológico aos profissionais de saúde através de redes sociais ou aplicativos para smartphones ${ }^{10}$ e a criação de um local para repouso dentro do hospital, evitando contaminação de familiares ou de outros no trajeto trabalho-casa ${ }^{11}$.

Nesta pandemia, se a necessidade da proteção dos profissionais dos estabelecimentos de saúde ganhou merecido destaque, o mesmo não se verifica para outros grupos ocupacionais. No Brasil, gráficos apresentando dados estratificados por sexo, faixa etária e região geográfica são elaborados com frequência como subsídios para orientar medidas de controle e prevenção, assim como para o planejamento e alocação dos recursos necessários para operar os sistemas de saúde. No entanto, na publicação dessas estatísticas, os microindicadores de morbidade não são desagregados até o nível da ocupação, o que não permite avaliar se, onde e em que circunstâncias os indivíduos testados positivos ou diagnosticados com a doença estavam trabalhando. Tampouco possibilita identificar focos de disseminação relacionados com atividades de trabalho.

A alta transmissibilidade do vírus, a grande proporção de infectados oligossintomáticos ou assintomáticos, estimada em mais de $30 \%^{12}$, a inexistência de vacina e de terapia medicamentosa comprovada, a insuficiente cobertura de testes, a duração prolongada dos quadros clínicos e as experiências de outros países explicam as decisões que provocaram as medidas de isolamento social e que determinaram que só os serviços essenciais fossem mantidos. Vale lembrar que, nesse contexto, para algumas categorias, houve intensificação das tarefas.

A preservação da saúde desses grupos essenciais, e de outros que se mantêm trabalhando por circunstâncias socioeconômicas, é fundamental para controlar a disseminação da doença e para a manutenção das pessoas em isolamento, confinamento ou quarentena, assim como para a atuação do próprio serviço de saúde e das demais atividades essenciais. Medidas preventivas são necessárias para atividades com maior risco de exposição ${ }^{13}$, como é o caso dos trabalhadores da farmácia, entregadores (delivery), carteiros, trabalhadores do transporte de cargas e de passageiros e pessoal de apoio, frentistas de postos de combustíveis, serviços de abastecimento e vendas de alimentos e de produtos; serviços residenciais, porteiros e zeladores, pessoal de limpeza, empregados(as) domésticos(as); vigilantes, policiais, bombeiros; cuidadores de idosos e de pessoas dependentes; de manutenção de serviços públicos e privados de telefonia, eletricidade, água, gás, internet, segurança pública, serviços funerários e coleta de lixo; e provavelmente outras atividades aqui não mencionadas.

Toda atividade de trabalho e todo trabalhador tem de ser considerado, e preparado, não apenas para a sua proteção, mas também para entender que sua atividade pode ter um papel importante no combate à epidemia. A exemplo de medidas adotadas por outros países ${ }^{14}$, uma ação coordenada com esse propósito poderia ser uma estratégia importante, como o devido treinamento das equipes de Saúde da Família do Sistema Único de Saúde que, se ampliadas, ainda têm como vantagem adicional o combate ao desemprego ${ }^{15}$. Comissões internas de prevenção de acidentes (CIPA) ou trabalhadores especialmente designados também podem ser preparados para atuar na prevenção da exposição ao vírus dentro das organizações.

Para assegurar condições laborais que propiciem redução na transmissão do vírus, medidas organizacionais necessitam ser discutidas no âmbito de cada atividade de trabalho e a práxis da Saúde do Trabalhador ${ }^{16}$ tem de ser considerada no rol das medidas e ações de saúde pública voltadas ao controle da pandemia. Dispomos de arcabouço jurídico robusto que sustenta a ação pública, suas políticas e regulamentações ${ }^{17-19}$, assim como ampla articulação e capilaridade no território nacional por meio dos programas e serviços de Saúde da Família e de Saúde do Trabalhador. Em seu conjunto, são ações articuladas nacionalmente e defendidas por organismos sindicais e instâncias do aparelho de Estado.

O planejamento e a tomada de decisões por autoridades responsáveis, com base em informações científicas, transparência e integração de ações, têm a ganhar se incorporadas as dimensões do trabalho, fator estruturante da nossa sociedade.

\section{Referências}

1. Baker MG, Peckham TK, Seixas NS. Estimating the burden of United States workers exposed to infection or disease: a key factor in containing risk of COVID-19 infection. medRxiv. 2020 [citado em 7 abr 2020]. Disponível em: https://doi.org/10.1101/2020.03.02.20030288

2. Li Q, Guan X, Wu P, Wang X, Zhou L, Tong Y, et al. Early transmission dynamics in Wuhan, China, of novel coronavirus-infected pneumonia. N Engl J Med. 2020 [citado em 4 abr 2020];382:1199-207. Disponível em : https://doi.org/10.1056/NEJMoa2001316 
3. Koh D. Occupational risks for COVID-19 infection. Occup Med. 2020 [citado em 4 abr 2020];70(1):3-5. Disponível em : https://doi.org/10.1093/occmed/kqaa036

4. BBC News Brasil [Internet]. São Paulo: Vinícius Lemos; 2020 [citado em 5 abr 2020]. Ministério Público do Trabalho analisa morte de doméstica no RJ após patroa ter coronavírus; [aproximadamente 2 telas]. Disponível em: https://www.bbc.com/portuguese/brasil-51982465

5. World Health Organization [Internet]. Genebra; 2020 [citado em 5 abr 2020]. Coronavirus disease (COVID-19) outbreak: rights, roles and responsibilities of health workers, including key considerations for occupational safety and health; [aproximadamente 1 tela]. Disponível em: https://www.who.int/publications-detail/ coronavirus-disease-(covid-19)-outbreak-rights-roles-and-responsibilities-of-health-workers-including-keyconsiderations-for-occupational-safety-and-health

6. Brasil. Ministério da Saúde. Secretaria de Atenção Especializada à Saúde. Departamento de Atenção Hospitalar, Domiciliar e de Urgência. Coordenação-Geral de Urgência. Força Nacional do Sistema Único de Saúde. Protocolo de manejo clínico para o novo coronavírus (2019-nCoV) [Internet]. Brasília (DF); 2020 [citado em 5 abr 2020]. Disponível em: http://189.28.128.100/dab/docs/portaldab/documentos/20200330_ ProtocoloManejo_ver06_Final.pdf

7. Associação Médica Brasileira [Internet]. São Paulo; 2020 [citado em 5 abr 2020]. Faltam EPIs em todo o país; [aproximadamente 2 telas]. Disponível em: https://amb.org.br/epi/

8. Morrison LG, Yardley L. What infection control measures will people carry out to reduce transmission of pandemic influenza? A focus group study. BMC Public Health. 2009 [citado em 7 abr 2020];9:258. Disponível em: https://doi.org/10.1186/1471-2458-9-258

9. Desclaux A, Sow K. «Humaniser » les soins dans l'épidémie d'Ebola ? Les tensions dans la gestion du care et de la biosécurité dans le suivi des sujets contacts au Sénégal. Anthropologie et Sante. 2015 [citado em 7 abr 2020];11. Disponível em : https://journals.openedition.org/anthropologiesante/1751\#quotation

10. Liu S, Yang L, Zhang C, Xiang YT, Liu Z, Hu S, Zhang B. Online mental health services in China during the COVID-19 outbreak. Lancet Psychiatry. 2020 [citado em 7 abr 2020];7(4):PE17-E18. Disponível em: https://doi.org/10.1016/S2215-0366(20)30077-8

11. World Health Organization [Internet]. Genebra; 2020 [citado em 5 abr 2020]. Getting your workplace ready for COVID-19; [aproximadamente 2 telas]. Disponível em: https://www.who.int/docs/default-source/coronaviruse/ getting-workplace-ready-for-covid-19.pdf?sfvrsn=359a81e7_6

12. Nishiura H, Kobayashi T, Miyama T, Suzuki A, Jung SM, Hayashi K, et al. Estimation of the asymptomatic ratio of novel coronavirus infections (COVID-19). Int J Infect Dis. 2020 [citado em 7 abr 2020];S1201-9712(20):1-7. Disponível em: https://doi.org/10.1016/j.ijid.2020.03.020

13. U. S. Department of Labor. Occupational Safety and Health Administration (Osha) [Internet]. USA; 2020 [citado em 5 abr 2020]. Guidance on preparing workplaces for COVID-19. Disponível em: https://www.osha.gov/Publications/OSHA3990.pdf

14. STAT [Internet]. Boston: James O’Donovan; 2020 [citado em 5 abr 2020]. Community health workers will be the main defense in rural Uganda against coronavirus; [aproximadamente 2 telas]. Disponível em: https://www.statnews.com/2020/03/28/community-health-workers-lead-covid-19-fight-uganda/

15. World Economic Forum [Internet]. Genebra; 2020 [citado em 5 abr 2020]. The job description for a COVID-19 community health worker - and how this could fight US unemployment; [aproximadamente 2 telas]. Disponível em: https://www.weforum.org/agenda/2020/03/retraining-unemployed-fight-covid-19/

16. Plataforma RENAST Online [Internet]. [citado em 5 abr 2020]. Saúde do Trabalhador; [aproximadamente 3 telas]. Disponível em: https://renastonline.ensp.fiocruz.br/temas/saude-trabalhador

17. Brasil. Decreto ${ }^{\circ}$ 7.602, de 7 de novembro de 2011: dispõe sobre a Política Nacional de Segurança e Saúde no Trabalho - PNSST. Diário Oficial da União [Internet]. 8 nov 2011 [citado em 5 abr 2020]. Disponível em: http://www.planalto.gov.br/ccivil_03/_ato2011-2014/2011/decreto/d7602.htm

18. Brasil. Ministério da Saúde. Portaria $\mathrm{n}^{0}$ 1.823, de 23 de agosto de 2012: institui a Política Nacional de Saúde do Trabalhador e da Trabalhadora. Diário Oficial da União [Internet]. 2012 [citado em 5 abr 2020]. Disponível em: https://bvsms.saude.gov.br/bvs/saudelegis/gm/2012/prt1823_23_08_2012.html

19. Brasil. Ministério da saúde. Secretaria da Gestão do Trabalho e da Educação na Saúde. Mesa Nacional de Negociação Permanente do SUS. Protocolo n ${ }^{\circ}$ 008/2011: protocolo da Mesa Nacional de Negociação Permanente do Sistema Único de Saúde - MNNP - SUS. Brasília, DF; 2011 [citado em 5 abr 2020].

Disponível em: https://portalarquivos2.saude.gov.br/images/pdf/2014/marco/10/protocolo08.pdf 
No editorial "A saúde do trabalhador e o enfrentamento da COVID-19", com número de DOI: 10.1590/2317-6369ED0000120, publicado no periódico Revista Brasileira de Saúde Ocupacional, 45:e14, na página $1 / 3$, corrige-se:

No último sobrenome do primeiro autor

\section{Onde se lia:}

"Fiho"

Leia-se:

"Filho" 